\title{
«Tarifs 2020 - Ignorer la réalité des coûts?»
}

\author{
Le développement des tarifs ambulatoires jusqu'en 2020, et tout particulièrement \\ l'incompatibilité entre la mise sur pied de tarifs appropriés et la neutralité des \\ coûts, était au centre de la 11e Journée des délégués tarifaires du 12 novembre 2014.
}

\section{Marina Lüscher, Christian Oeschger}

FMH, Division Tarifs et conventions pour la médecine ambulatoire en Suisse
Correspondance: FMH, Division Tarifs et conventions pour la médecine ambulatoire en Suisse Frohburgstrasse 15 CH-4600 Olten Tél. 0313591230 Fax 0313591238
Le Dr Ernst Gähler, vice-président de la FMH, a tout d'abord souhaité la bienvenue aux plus de 100 participants à la deuxième Journée des délégués tarifaires de cette année, organisée par le département Tarifs et conventions pour la médecine ambulatoire en Suisse. Après une brève introduction, il a cédé la parole à la Dresse Yvonne Gilli, conseillère nationale et membre de la Commission de la sécurité sociale et de la santé publique. Dans son exposé, intitulé «Ouvrons l'œil! La Berne fédérale porte des œillères», Y. Gilli a pris position sur l'intervention du Conseil fédéral dans les tarifs le $1^{\text {er }}$ octobre 2014, plusieurs modifications concernant le laboratoire au cabinet ainsi que sur les modifications prévues dans la loi sur les produits thérapeutiques, qui revêtent une grande importance aux yeux du corps médical.

Puis, le Dr Ernst Gähler a présenté la situation actuelle dans le domaine des tarifs ambulatoires. L'équipe du vice-président de la FMH se penche actuellement notamment sur l'intervention du Conseil fédéral dans les tarifs, le laboratoire au cabinet, la future organisation de la procédure EAE et la réorganisation de la société TARMED Suisse SA, mais l'un des principaux projets reste la révision globale de la structure tarifaire TARMED.

Le fait que la Confédération soit confrontée à un dilemme entre la structure et le prix a également un impact sur les travaux actuels, évoqués par le Dr Ernst Gähler, dans le cadre de la révision du TARMED. Conrad Engler, membre de la direction de $\mathrm{H}+$, n'a du reste pas hésité à qualifier cette révision de «nœud gordien» lors de son exposé qui s'est terminé par la conclusion suivante: «L'autonomie tarifaire avance à la vitesse des rameurs, et la Confédération donne le rythme.» Ernst Gähler voit également une contradiction entre la stratégie de la Confédération, telle qu'elle est décrite dans la stratégie «Santé2020», et les coûts réels. Pour lui, il est clair que la Confédération ne veut pas voir la réalité des coûts.

Après avoir échangé leurs impressions pendant la pause, les participants ont eu la possibilité d'assister à l'une des trois séances parallèles.

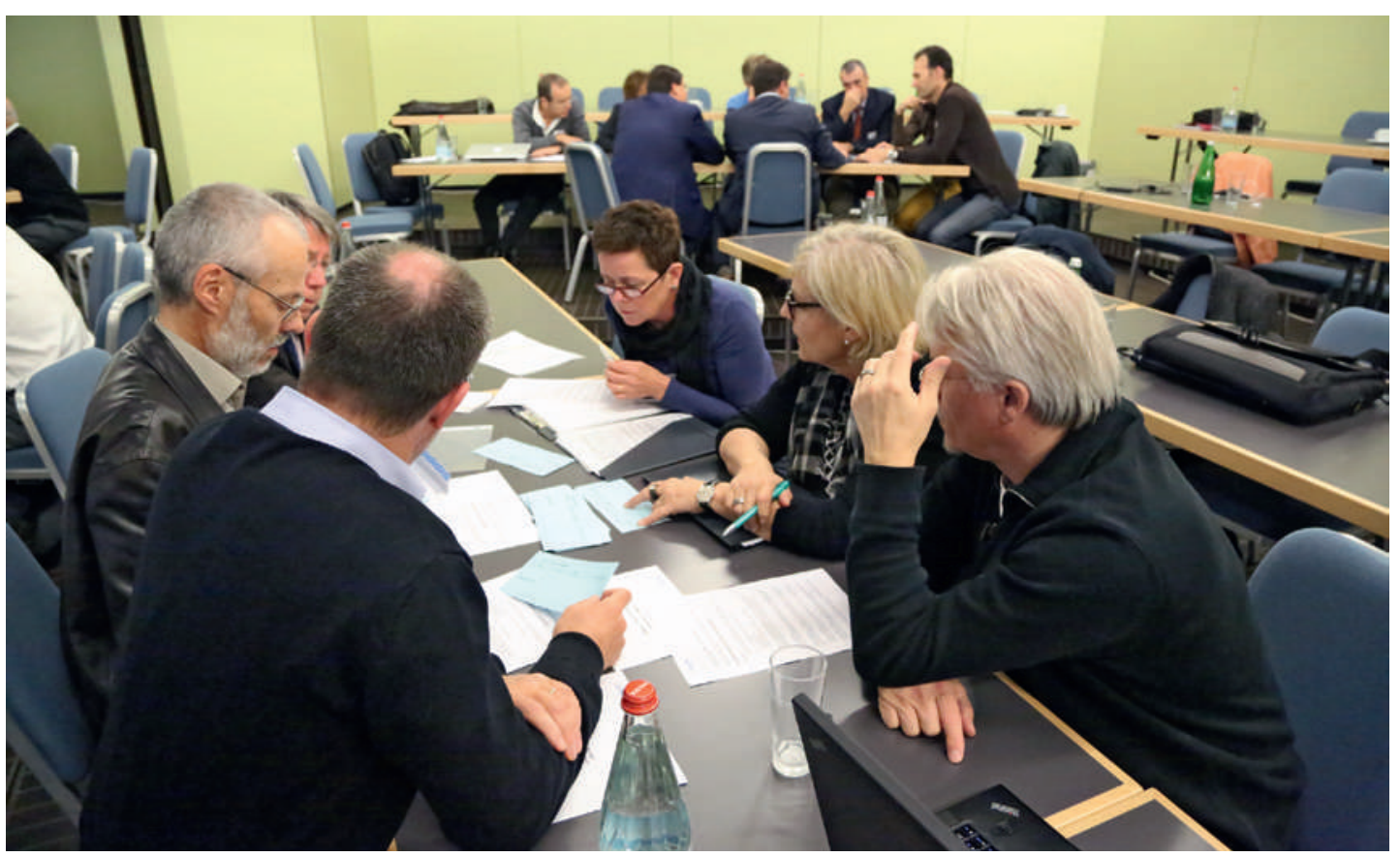

Les séances parallèles ont permis aux participants d'échanger leurs expériences entre confrères. 


\section{Séances parallèles}

Séance parallèle 1 :

\section{Les bases du système tarifaire TARMED}

Lors de cet atelier, les délégués tarifaires ont pu se familiariser avec le TARMED ainsi qu'avec ses bases légales, contractuelles et techniques. Ils ont ensuite mis en pratique leurs connaissances en matière de saisie de prestations à l'occasion d'un exercice basé sur des exemples de cas tirés du TARMED. Pour terminer, une partie importante de l'atelier a été consacrée aux questions, afin de favoriser l'échange entre les délégués.

\section{Séance parallèle 2: De la prestation à la} rémunération - le long parcours d'une facture «Pouvez-vous expliquer toutes les informations qui se trouvent sur le formulaire de facturation standardisé?» Le petit quiz réalisé en début de séance a montré que même les médecins ont parfois de la peine à expliquer tous les paramètres du formulaire. Anton Prantl, directeur exécutif de la Caisse des médecins, a ensuite présenté quelques faits et chiffres intéressants autour de la facture. Saviez-vous par exemple que près de $3 \%$ des factures ambulatoires sont payées en double? Sonja Aerne et Wolfram Strüwe ont quant à eux proposé un aperçu du traitement des factures chez Helsana, qui en contrôle, comptabilise et rembourse plus de 14 millions par an. Les participants ont profité de l'occasion pour poser de nombreuses questions.

Séance parallèle 3: La révision tarifaire en pratique: productivité des médecins et critères relatifs aux patients dans le TARMED - un défi tarifaire Pour le Dr Beat Dubs, président de la task force TARMED de la SSUM, le nombre de «cas probléma- tiques» a augmenté ces dernières années dans les cabinets par rapport au début de sa carrière. Aujourd'hui, les consultations durent plus longtemps, les collaborateurs sont plus souvent absents, les tâches administratives explosent et les patients ont des attentes toujours plus élevées. L'objectif de cet atelier a été de montrer comment le TARMED tient compte d'une part de l'hétérogénéité des patients et, de l'autre, du fait que les médecins doivent également suivre des cours de formation continue ou remplir des statistiques, des tâches qui ne sont pas directement rémunérées par le tarif. Lors des discussions, en groupe puis en plénum, les participants ont fait preuve d'une grande sensibilité pour ce thème mais ont également montré que la productivité médicale et les critères relatifs aux patients ont tout à fait leur place dans la révision en cours du TARMED.

\section{Le corps médical entre régulation des admissions et liberté de contracter}

Lors de son exposé, le Dr Jürg Schlup, président de la $\mathrm{FMH}$, a rappelé que la grande majorité du Conseil national a approuvé la motion Stahl le 10 septembre 2014. Selon la FMH, qui s'est exprimée dans le Tages-Anzeiger le 12 septembre 2014, cette version «allégée» de la liberté de contracter est trop «précipitée». Jusqu'ici, il n'a pas été possible de démontrer que la clause du besoin a permis d'économiser des coûts. Avec cette version «allégée», on ne fait que retourner à un système qui n'a jamais fait ses preuves, selon J. Schlup, par ailleurs convaincu que cette solution est encore pire qu'un statu quo. C'est pourquoi la FMH demande que les médecins qui ont exercé au moins trois ans dans un hôpital suisse et qui maîtrisent une langue nationale en soient exemptés.

Dans son exposé, Roger Scherrer, chef de la division Tarifs et conventions pour la médecine ambula-

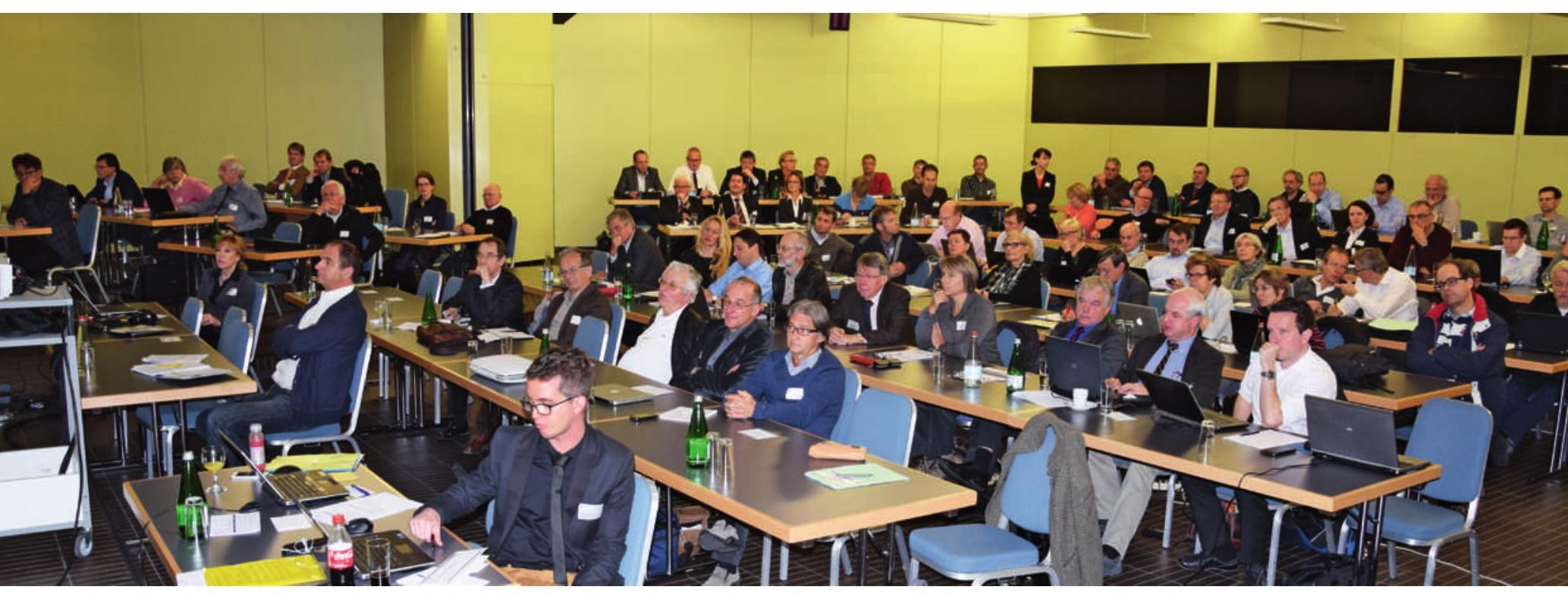

Avec plus de 100 participants, la Journée des délégués tarifaires de novembre 2014 a une nouvelle fois connu un grand succès. 


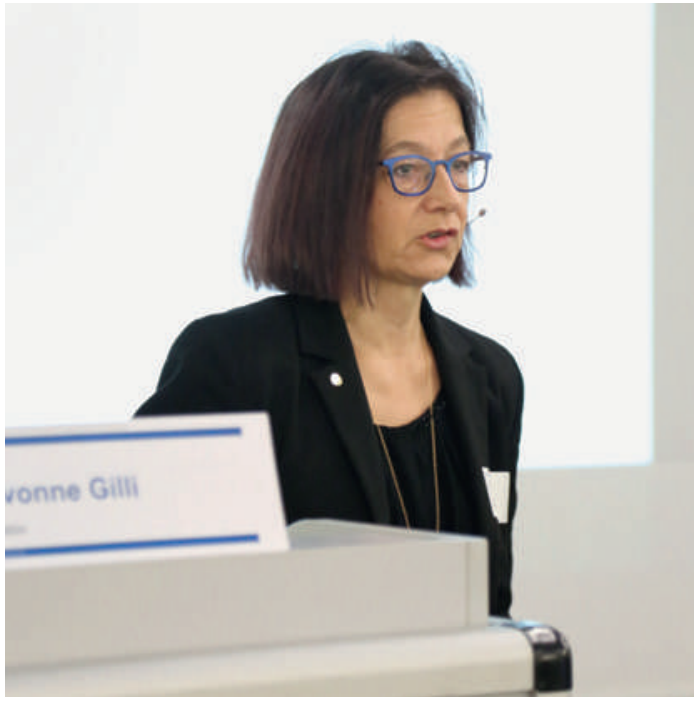

La conseillère nationale Yvonne Gilli a abordé les modifications prévues concernant la loi sur les produits thérapeutiques.

toire en Suisse, a fait le point sur l'avancement des travaux dans le cadre de la révision du TARMED. Lors du dernier semestre, de nouvelles équipes spécialisées

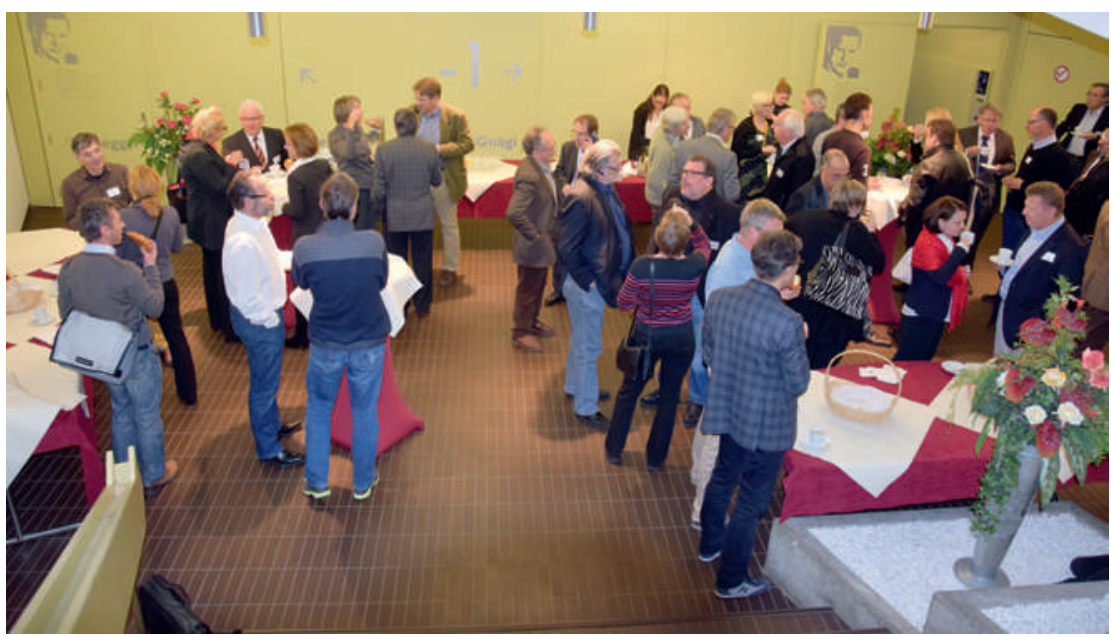

Premières discussions autour d'un café et d'un croissant. se sont constituées. Les équipes consacrées à la cardiologie et à la chirurgie de la main ont d'ores et déjà débuté la phase-pilote et les premiers entretiens entre experts ont eu lieu. Entretemps, plus de 20 équipes spécialisées sont à l'œuvre.

La Dresse Heidi Zinggeler Fuhrer et le Dr Rolf Temperli ont quant à eux présenté le point de vue des médecins de famille et des pédiatres sur la révision tarifaire. Pour revaloriser leurs disciplines, ils demandent l'adaptation du revenu de référence des médecins, un calcul correct des unités fonctionnelles ainsi que des minutages et des paramètres de productivité corrects. Les médecins de famille sont convaincus qu'ensemble, ils parviendront à leur objectif.

«2020: le TARMED sera-t-il dépassé?» C'est la question posée par Otto Bitterli, CEO de Sanitas Assurance Maladie. Avec la numérisation accrue que nous connaissons d'ores et déjà dans d'autres secteurs, la relation entre médecin et patient est en pleine évolution. Les assureurs ont conscience de ce changement de paradigme et se sont fixé comme objectif d'étudier ensemble les nouvelles possibilités en utilisant la marge de manœuvre à disposition. C'est la raison pour laquelle, selon Otto Bitterli, les problèmes liés au TARMED doivent être résolus le plus vite possible. Il s'agit de créer une confiance mutuelle et d'encourager la collaboration.

Avant de terminer la journée autour d'un apéro, Ernst Gähler revient sur les principaux moments en soulignant encore une fois l'importance de la révision du TARMED. Même si les conditions-cadres ne sont pas toujours optimales, nous avons tout de même remporté plusieurs succès. Dans cet esprit, à nous de maintenir l'autonomie tarifaire!

Le département Tarifs et conventions pour la médecine ambulatoire en Suisse tient à remercier tous les orateurs et participants pour leur engagement et les discussions constructives. Sur le site internet de la FMH vous trouverez de plus amples informations ainsi que les transparents des exposés et des séances parallèles (www.fmh.ch $\rightarrow$ Tarifs ambulatoires $\rightarrow$ Journée des délégués tarifaires). 